\title{
Pulmonary Tumor Embolism Secondary to Uterine Corpus Carcinosarcoma Mimicking Pulmonary Thromboembolism
}

\author{
Makoto Hibino, Kenichiro Akazawa, Koji Hikino and Motoki Oe
}

\begin{abstract}
We herein report a case of pulmonary tumor embolism caused by hematogenous metastasis that mimicked pulmonary thromboembolism in a 62-year-old Japanese woman with a history of uterine corpus carcinosarcoma. The case suggests that tumor embolism must be included in the differential diagnoses of respiratory symptoms in patients with a history of malignancy. It also illustrates the usefulness of such findings as beaded, dilated pulmonary arteries by computed tomography (CT) and high ${ }^{18} \mathrm{~F}$-fluorodeoxyglucose (FDG) uptake by fusion FDG positron emission tomography/CT imaging for differentiating a pulmonary tumor embolism from pulmonary thromboembolism.
\end{abstract}

Key words: pulmonary tumor embolism, pulmonary tumor thrombotic microangiopathy, uterine corpus carcinosarcoma, beaded, dilated pulmonary artery, ${ }^{18} \mathrm{~F}$-fluorodeoxyglucose positron emission tomography

(Intern Med 51: 2603-2607, 2012)

(DOI: 10.2169/internalmedicine.51.7220)

\section{Introduction}

The differential diagnoses of respiratory symptoms in patients with a history of malignancy include infection, lung metastasis, and lymphangitic carcinomatosis. Patients with malignancy sometimes develop hypercoagulopathy, deep venous thrombosis, and pulmonary thromboembolism. In general, pulmonary embolism is a type of thromboembolism; however, the emboli may be either thrombotic, or less frequently, nonthrombotic. Rarely, tumor cells may invade and occlude the pulmonary arteries and induce a pulmonary tumor embolism. This condition often develops as pulmonary tumor thrombotic microangiopathy (PTTM) and rarely as a massive embolism within the proximal pulmonary artery. We herein report a case of tumor embolism caused by hematogenous metastasis of uterine corpus carcinosarcoma that was detected by computed tomography (CT) findings of beaded, dilated pulmonary arteries and fusion ${ }^{18} \mathrm{~F}$-fluorodeoxyglucose positron emission tomography/CT (FDG PET/CT) imaging showing a high FDG uptake.

\section{Case Report}

A 62-year-old Japanese woman with a history of radical hysterectomy for carcinosarcoma of the uterine corpus, with pathological staging of T1cN0M0, was admitted with a cough and hemoptysis 28 months postoperatively. She had developed a pulmonary embolism 18 months postoperatively and had been treated with warfarin. Chest CT scans revealed pulmonary emboli in the right main pulmonary artery and the middle and lower lobar arteries. The patient also had a history of type 2 diabetes and took $1 \mathrm{mg}$ glimepiride daily.

On physical examination, the patient was not in acute distress. Her vital signs were within the normal limits. Positive findings included an increased intensity of P2 and mild dilatation of the jugular vein. Initial laboratory tests revealed that the prothrombin time-international normalized ratio (PTINR) was 1.36 and the D-dimer level was $1.01 \mu \mathrm{g} / \mathrm{mL}$. Ultrasound cardiography showed mild dilatation of the right atrium and ventricle, and a slightly high estimated pulmonary artery systolic pressure $(41 \mathrm{mmHg})$. Contrast-enhanced chest CT scans showed filling defects within the right main pulmonary artery and the middle and lower lobar arteries 

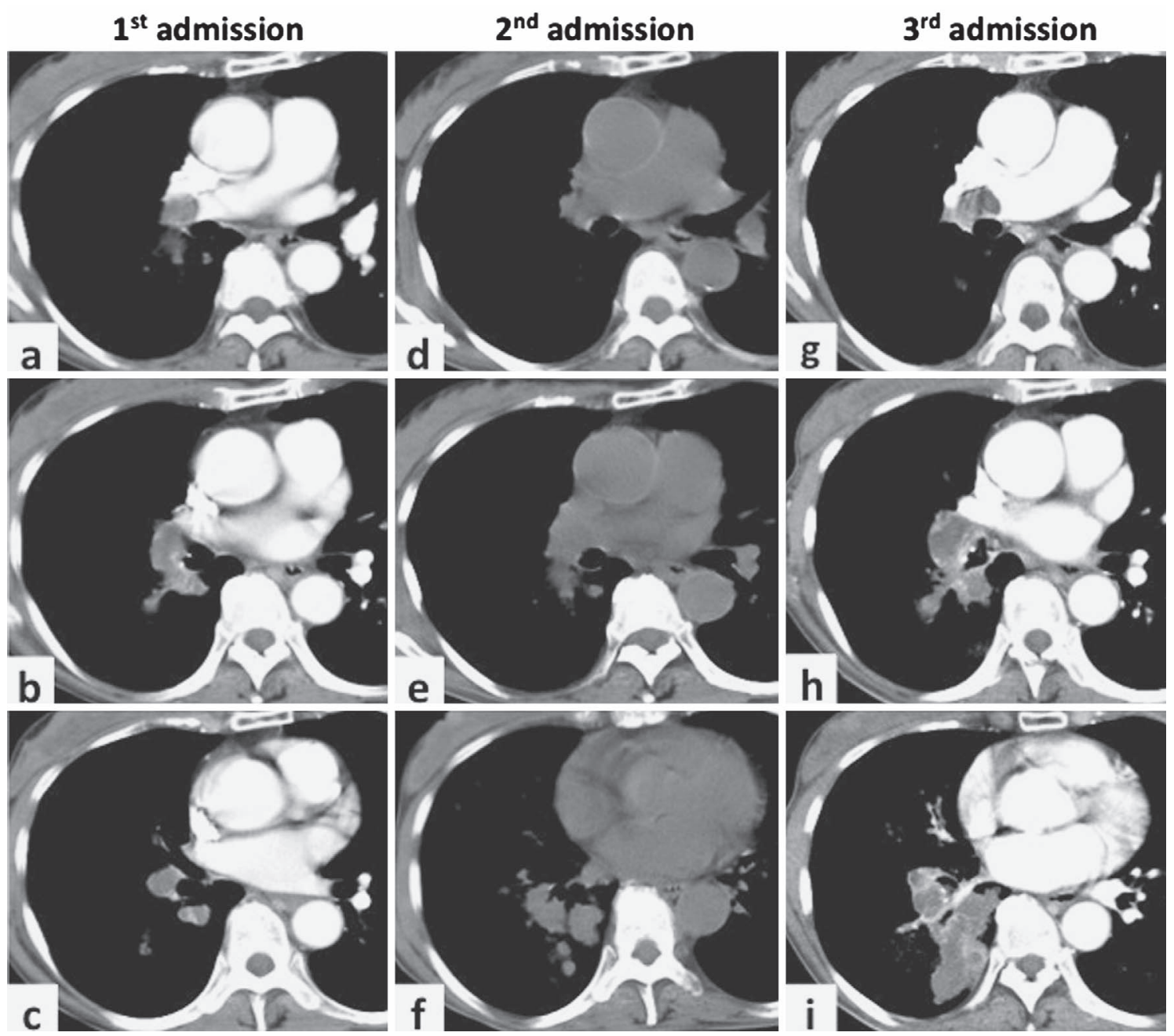

Figure 1. Chest CT scans at the first admission (a-c) showed filling defects within the right main pulmonary artery, as well as the middle and lower lobar arteries in the right lung. CT scans at the second (d-f) and third (g-i) admissions showed beaded, dilated pulmonary arteries corresponding to the intraluminal filling defects. The mass on the dorsal side of the right lower lobe was connected to the main pulmonary artery, and was thought to be a branch of the abnormally distended pulmonary $\operatorname{artery}(\mathbf{i})$.

(Fig. 1a-c)). A technetium-99 m macroaggregated albumin $\left({ }^{99 m}\right.$ Tc-MAA) perfusion scan showed a lack of perfusion in the right middle and lower lobes. Deep venous thrombosis was not detected by Doppler ultrasound.

We considered the hemoptysis to be associated with pulmonary thromboembolism. After the hemoptysis had stopped, the patient was discharged when the PT-INR increased to the therapeutic range (post-admission day 7). Two months after the first admission, the patient returned to the emergency department because of hemoptysis. She had respiratory failure with an oxygen saturation (digital pulse oximetry) of $88 \%$ while breathing ambient air at rest and a respiratory rate of 24 breaths per minute. Chest CT scans revealed a ground-glass shadow with centrilobular distribution especially in the right lower lobe (lung window, not shown), suggesting aspiration of blood, and a beaded and mildly dilated pulmonary artery was noted between the right main pulmonary artery and the middle and lower lobar arteries (mediastinal window; Fig. 1d-f).
Bronchoscopy, performed after the anticoagulation therapy was stopped, did not reveal the source of bleeding. Observation alone showed improved infiltration on her chest radiograph and ameliorated respiratory conditions. We thought that this desaturation had been caused by an alveolarcapillary block with blood aspiration, and not by a V/Q mismatch with progression of pulmonary tumor embolism. After the hemoptysis and respiratory failure improved, anticoagulation therapy was reinitiated, and the patient was discharged and scheduled for follow-up bronchoscopy in the outpatient clinic.

Two months after the second admission, the patient again developed hemoptysis. Contrast-enhanced chest CT scans revealed beaded and obviously dilated pulmonary arteries in the lower lobe (Fig. 1g-i). We therefore suspected a pulmonary tumor embolism. Fusion FDG PET/CT imaging showed high FDG uptake (Fig. 2a) at the sites of the pulmonary embolism, thus suggesting it to be a pulmonary tumor embolism. 

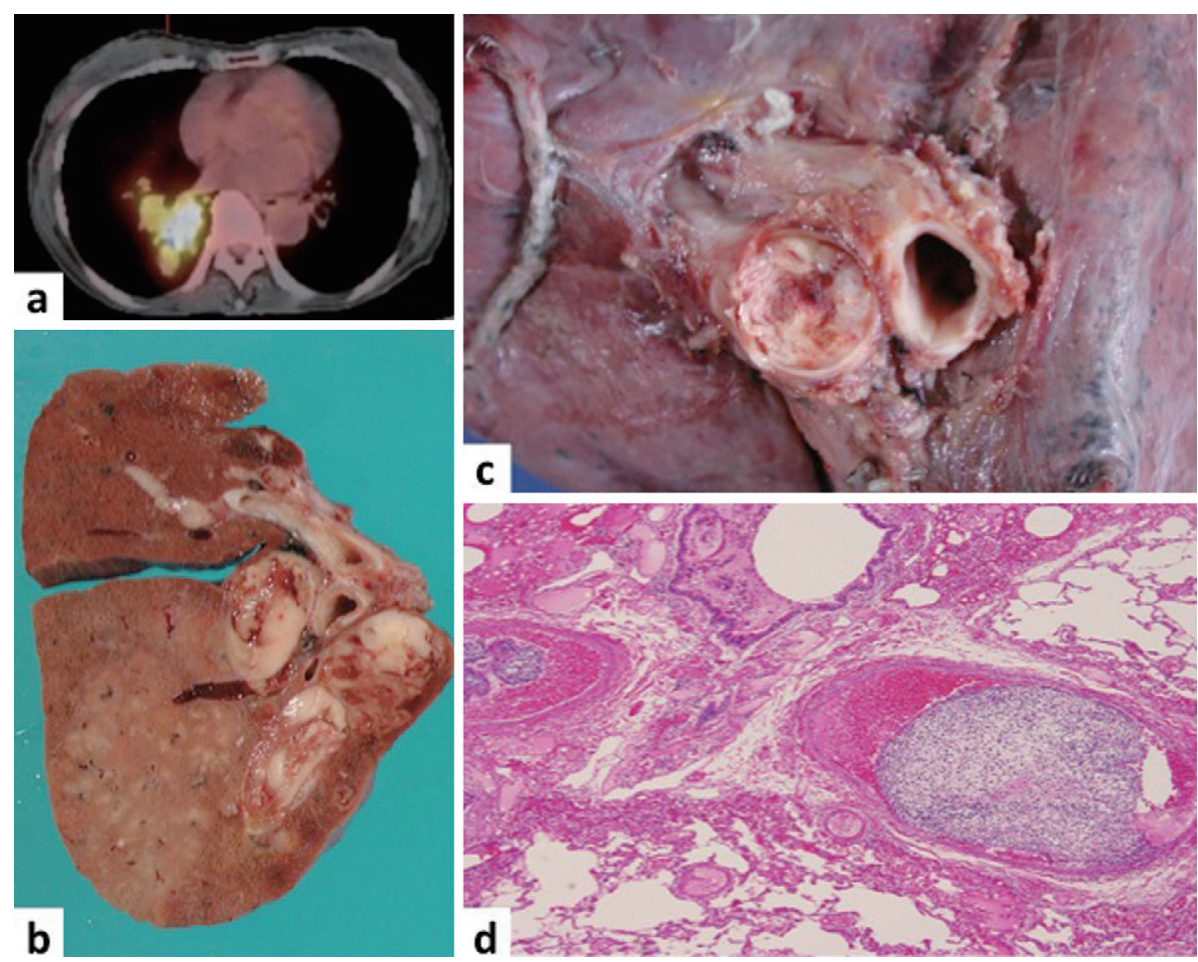

Figure 2. Fusion FDG PET/CT imaging showed high FDG uptake (maximum standard uptake value, 9.0) at the sites of embolism (a). A macroscopic examination of the resected middle and lower lobes of the right lung showed dilatation of the lower and middle lobar arteries (b) and distension of the right main pulmonary artery (c), with an endoluminal yellow-white tumor embolus. A histopathological examination revealed that the vascular lumens were occluded mainly by tumor cells (d).

The patient underwent a right middle and lower lobectomy. The resected specimens of the right middle and lower lobes showed a distended right main pulmonary artery and obviously dilated lower and middle lobar arteries (Fig. 2b), with an endoluminal yellow-white tumor embolus (Fig. 2b, c). A histopathological examination revealed that the lumens of numerous small pulmonary arteries were filled with tumor cells (Fig. 2d) consistent with carcinosarcoma of the uterine corpus. Lymph node metastasis, generalized lymphatic dissemination, and pulmonary infarction were absent.

After the lobectomy, we proposed adjuvant chemotherapy; however, the patient chose only observation. Her condition deteriorated over time, and 12 months after the lobectomy, she died of multiple lung metastases and respiratory failure.

\section{Discussion}

Uterine corpus carcinosarcoma, also known as malignant mixed Mullerian tumor, is an uncommon neoplasm with a poor prognosis. Advanced or recurrent cases are associated with an especially poor prognosis, despite surgical resection and chemotherapy (1).

Pulmonary thromboembolism is difficult to diagnose by chest radiography, because normal findings are usually obtained. Its clinical symptoms and severity vary on a case-bycase basis: it may range from asymptomatic disease to death by hemodynamic collapse. The treatment for this condition depends on the severity of the hemodynamic state, and in- cludes anticoagulant therapy, thrombolytic therapy, and medical or surgical embolectomy (2-4). However, in patients with a history of malignancy, an embolism may be caused by not only thrombi, but also tumor cells (5-7).

Enhanced chest CT does not enable differentiation between pulmonary thromboembolism and pulmonary tumor embolism, because it often reveals only filling defects in the pulmonary arteries $(8,9)$. Further, anticoagulant and thrombolytic therapies are not fundamental, but only supportive, for pulmonary tumor embolism. Therefore, alternative treatments such as resection, chemotherapy, or radiation therapy for the malignancy itself should be considered for the management of pulmonary tumor embolism.

Kane et al. (10) and Winterbauer et al. (11) described four basic types of pulmonary vascular involvement by tumor emboli: (i) large tumor emboli occluding either the main pulmonary arteries or the large lobar and segmental branches, which can produce acute pulmonary hypertension; (ii) pure microscopic tumor emboli involving the small arteries or arterioles, which often cause progressive dyspnea and subacute pulmonary hypertension; (iii) pulmonary microvascular invasion as a part of generalized lymphatic dissemination (lymphangitic carcinomatosis), which may lead to diffuse interstitial infiltrates; (iv) and combinations of these three mechanisms. Another type of pulmonary tumor embolism is known as PTTM, a term proposed by von Herbay et al. (12). Tumor cells may progress from lymphatic ducts, via the thoracic lymph duct and superior vena cava, 
to the pulmonary circulation. In the early stage, tumor cells occlude small pulmonary arteries. Such obstruction causes cell-mediated immune responses, which induce medial hypertrophy with cellular intimal proliferation and thrombosis, leading to narrowing and occlusion the vascular lumens. Within a few days, pulmonary hypertension, cor pulmonale, or death may occur.

Although many cases of PTTM have been reported, tumor embolism of a large, proximal pulmonary artery has rarely been described (11-13). Further, because the prevalence of tumor embolism of a pulmonary artery is low, an antemortem diagnosis is rare. PTTM has been found during autopsy in approximately $0.9-3.3 \%$ of patients with malignant tumors (14). As a tumor embolus in a large pulmonary artery is rarely diagnosed either antemortem or postmortem, its frequency is unknown.

In the present case, the pulmonary embolism was detected 18 months after the operation. Proximal embolization with a tumor is often accompanied by the acute onset of symptoms and invasion of the vena cava by the primary tumor (11). However, in this patient, neoplastic invasion of the vena cava and acute onset of symptoms were not observed. An acute, large pulmonary embolism is considered to be symptomatic condition. However, our patient did not show symptoms of embolism during the entire course of the study, and autopsy revealed that the embolus in the pulmonary artery was mainly composed of tumor cells. Therefore, it is unlikely that a pulmonary thromboembolism occurred first, followed by the deposition of tumor cells in the thrombus, which then progressed to the proximal end of the artery. Instead, we believe that the large asymptomatic tumor embolism developed because of the adhesion of tumor cells to the inner wall of the peripheral pulmonary artery, followed by their progression to the proximal end of the artery.

For the diagnosis of tumor embolism of a large, proximal pulmonary artery, the following findings are necessary: a beaded, dilated pulmonary artery on CT scans (15), high FDG uptake corresponding to the sites of embolism on FDG PET/CT images (16-18), and the presence of tumor cells by cytology with right heart catheterization (19). Among these tests, the latter two are performed only when tumor embolism is suspected. Recently, enhanced chest CT has been considered the gold standard for diagnosing pulmonary embolism. Clinical suspicion of tumor embolism is important so that the latter two tests will be performed, and so that the pulmonary tumor embolism can be differentiated from a pulmonary thromboembolism. Therefore, a beaded, dilated pulmonary artery on CT scans is the first important finding to diagnose pulmonary tumor embolism. This finding is very rare, and CT has low sensitivity. However, the finding is specific for tumor embolism; therefore, physicians should be familiar with it, especially as the sensitivity of CT continues to improve.

Second, FDG PET/CT imaging is useful to distinguish pulmonary tumor embolism from pulmonary thromboembolism. However, acute thromboembolism may also show high FDG uptake, which is caused by focal inflammation and necrosis of the intravascular wall, accompanied by aggregation of leukocytes on the endothelial surface $(20,21)$. In 13 cases of pulmonary thromboembolism, the mean standardized uptake value (SUV) of vessels without thrombi was $1.15 \pm 0.38$ (range, 0.42-1.64), and pulmonary thromboembolism had a slightly increased mean SUV of $1.65 \pm 0.61$ (range, 0.45-3.03) (21). On the other hand, reports have shown that pulmonary tumor emboli can have an obviously increased FDG metabolism: a $\mathrm{SUV}_{\max }$ of 7.8 was reported in a case of osteogenic sarcoma (16), a $\mathrm{SUV}_{\max }$ of 8.2 in a case of choriocarcinoma (17), and a $\mathrm{SUV}_{\max }$ of 7.3 in another case of choriocarcinoma (18). The FDG uptake is lower in acute pulmonary embolism than in pulmonary tumor embolism, which shows a high FDG uptake similar to a primary tumor. Therefore, a high FDG uptake area corresponding to the sites of embolism is indicative of tumor embolism.

Finally, right heart catheterization is needed to make a definite diagnosis. In cases of PTTM, pulmonary wedge aspiration cytology specimens can be obtained by placing a pulmonary artery catheter in a wedged position. In tumor embolism of a large, proximal pulmonary artery, aspiration cytology specimens can be obtained after embolectomy. However, a negative finding by pulmonary artery aspiration cytology does not exclude a diagnosis of pulmonary tumor embolism.

In conclusion, we could not diagnose the present case as pulmonary tumor embolism at the first admission, despite the use of chest CT, because tumor embolism of a large, proximal pulmonary artery is very rare and the arterial dilatation was mild in the early stage. This case suggests that pulmonary tumor embolism should be suspected in patients diagnosed with pulmonary embolism, especially after a history of malignancy. Furthermore, a beaded, dilated pulmonary artery on chest CT scans and high FDG uptake by FDG PET/CT imaging are valuable findings to diagnose tumor embolism.

\section{The authors state that they have no Conflict of Interest (COI).}

\section{Acknowledgement}

We thank Dr. Kazunao Watanabe for performing the lobectomy and Dr. Yoshio Miyazawa for evaluating the pathological findings.

\section{References}

1. Silverberg SG, Major FJ, Blessing JA, et al. Carcinosarcoma (malignant mixed mesodermal tumor) of the uterus. A Gynecologic Oncology Group pathologic study of 203 cases. Int J Gynecol Pathol 9: 1-19, 1990.

2. Wang C, Zhai Z, Yang Y, et al. Efficacy and safety of low dose recombinant tissue-type plasminogen activator for the treatment of acute pulmonary thromboembolism: a randomized, multicenter, controlled trial. Chest 137: 254-262, 2010.

3. Konstantinides S, Geibel A, Heusel G, Heinrich F, Kasper W. Management Strategies and Prognosis of Pulmonary Embolism-3 Trial Investigators. Heparin plus alteplase compared with heparin 
alone in patients with submassive pulmonary embolism. N Engl $\mathrm{J}$ Med 347: 1143-1150, 2002.

4. Goldhaber SZ. Advanced treatment strategies for acute pulmonary embolism, including thrombolysis and embolectomy. J Thromb Haemost 7: 322-327, 2009.

5. Rossi SE, Goodman PC, Franquet T. Nonthrombotic pulmonary emboli. AJR Am J Roentgenol 174: 1499-1508, 2000.

6. Wittram C, Maher MM, Yoo AJ, Kalra MK, Shepard JA, McLoud TC. CT angiography of pulmonary embolism: diagnostic criteria and causes of misdiagnosis. Radiographics 24: 1219-1238, 2004.

7. Jorens PG, Van Marck E, Snoeckx A, Parizel PM. Nonthrombotic pulmonary embolism. Eur Respir J 34: 452-474, 2009.

8. Chan CK, Hutcheon MA, Hyland RH, Smith GJ, Patterson BJ, Matthay RA. Pulmonary tumor embolism: a critical review of clinical, imaging, and hemodynamic features. J Thorac Imaging $\mathbf{2}$ : 4-14, 1987.

9. Roberts KE, Hamele-Bena D, Saqi A, Stein CA, Cole RP. Pulmonary tumor embolism: a review of the literature. Am J Med 115 228-232, 2003.

10. Kane RD, Hawkins HK, Miller JA, Noce PS. Microscopic pulmonary tumor emboli associated with dyspnea. Cancer 36: 14731482, 1975.

11. Winterbauer RH, Elfenbein IB, Ball WC Jr. Incidence and clinical significance of tumor embolization to the lungs. Am J Med 45: 271-290, 1968.

12. von Herbay A, Illes A, Waldherr R, Otto HF. Pulmonary tumor thrombotic microangiopathy with pulmonary hypertension. Cancer 66: $587-592,1990$.
13. Wong PS, Aye WM, Lee CN. Pulmonary tumor embolism secondary to osteosarcoma. Ann Thorac Surg 77: 341, 2004.

14. Sato Y, Marutsuka K, Asada Y, Yamada M, Setoguchi T, Sumiyoshi A. Pulmonary tumor thrombosis microangiopathy. Pathol Int 45: 436-440, 1995.

15. Shepard JO, Moore EH, Templeton PA, McLoud TC. Pulmonary intravascular tumor emboli: dilated and beaded peripheral pulmonary arteries at CT. Radiology 187: 797-801, 1993.

16. Purandare NC, Dua SG, Rangarajan V, Shah S, Sharma AR. Pulmonary artery and femoral vein tumour thromboembolism in a patient with osteogenic sarcoma demonstrated by FDG PET/CT. Eur J Nucl Med Mol Imaging 37: 653, 2010.

17. Zaheer S, Osmany S, Lai HK, Eng DN. Usefulness of F-18 fluorodeoxyglucose positron emission tomography/computed tomography in a case of choriocarcinoma presenting as pulmonary embolism. Clin Nucl Med 34: 343-345, 2009.

18. Sone T, Yoshikawa K, Fukunaga M. Pulmonary tumor embolism from choriocarcinoma: detection with F-18 FDG positron emission tomography. Clin Nucl Med 33: 773-774, 2008.

19. King MB, Harmon KR. Unusual forms of pulmonary embolism. Clin Chest Med 15: 561-580, 1994.

20. Goethals I, Smeets P, De Winter O, Noens L. Focally enhanced F18 fluorodeoxyglucose (FDG) uptake in incidentally detected pulmonary embolism on PET/CT scanning. Clin Nucl Med 31: 497498, 2006.

21. Wittram C, Scott JA. ${ }^{18}$ F-FDG PET of pulmonary embolism. AJR Am J Roentgenol 189: 171-176, 2007.

(C) 2012 The Japanese Society of Internal Medicine http://www.naika.or.jp/imonline/index.html 